\title{
Data breach o violazione dei datt?
}

\author{
Michele A. Cortelazzo
}

PUBBLICATO: 24 APRILE 2020

\section{Quesito:}

Ci è stato segnalato l'avviso con il quale l'INPS, nel proprio sito istituzionale, ha informato gli utenti di avere "prontamente notificato il data breach al Garante per la protezione dei dati personali". Chi ci ha scritto ha commentato: "il sottoscritto NON conosce l'inglese né è tenuto a conoscerlo in base a disposizioni o leggi attualmente in vigore" e ha invitato l'Accademia ad attivarsi "per contrastare, almeno nei siti istituzionali, l'ingiustificata e incomprensibile deriva anglofona che colpisce il nostro Paese, soprattutto in determinati ambienti dove l'uso dell'inglese stride con la necessità di un linguaggio comprensibile, chiaro e inclusivo".

\section{Data breach o violazione dei dati?}

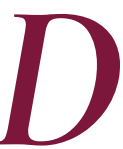

ata breach indica 'una violazione di sicurezza che comporta - accidentalmente o in modo illecito - la distruzione, la perdita, la modifica, la divulgazione non autorizzata o l'accesso ai dati personali trasmessi, conservati o comunque trattati'. La locuzione inglese data breach vale, letteralmente, proprio 'violazione dei dati', ed è usata prevalentemente, se non esclusivamente, in riferimento ai "dati personali" (spesso in senso ancora più restrittivo, in riferimento ai "dati personali sensibili"). In questo senso è in uso in inglese dai primi anni di questo secolo. L'ho trovata utilizzata, per esempio, nel "New York Times" a partire dall'aprile (e poi giugno) 2005, in riferimento a un caso fortuito di accesso a dati personali avvenuto nei pressi di Berlino e a un caso di appropriazione dei dati dei possessori di carte di credito, avvenuto negli Stati Uniti. Nell'anno successivo il tema (e, parallelamente, l'espressione) è stato oggetto di un disegno di legge sulla protezione dal furto dell'identità personale, discusso nel Senato federale ed è stato introdotto nei dibattiti di senati di singoli stati, in atti giurisprudenziali, in testi di dottrina giuridica.

In Europa l'espressione si è diffusa soprattutto a partire dal 20I6, quando i due organi che esercitano il potere legislativo dell'Unione Europea, il Parlamento e il Consiglio, hanno emanato il Regolamento (2016, n. 679) noto come "Regolamento generale sulla protezione dei dati" (o come GDPR, con la sigla basata sulla denominazione inglese). Le versioni nazionali hanno adottato soluzioni diverse per rendere personal data breach del testo inglese:

- alcune lingue sono ricorse all'equivalente violazione dei dati personali (oltre all'italiano, il portoghese violação de dados pessoais, ma anche, ad esempio, il croato povreda osobnih podataka);

- altre lingue hanno adottato una soluzione più estesa per l'inglese data, come il francese che utilizza violation de données à caractère personnel (segue questa soluzione il greco paravíasi dedoménon prosopikoú charaktíra);

- altre lingue ricorrono a un'espressione ancora più estesa, come lo spagnolo violaciones de la seguridad de los datos personales e il romeno incălcare a securității datelor cu caracter personal, che esplicitano il concetto di 'sicurezza', probabilmente sul modello del tedesco Verletzung des Schutzes personenbezogener Daten (letteralmente 'violazione della protezione dei dati personali'). 
Non c'è stata, quindi, una soluzione univoca, neppure all'interno delle lingue romanze. Tra le denominazioni scelte dalle diverse lingue, quella italiana si avvicina, più delle altre, alla forma inglese, per il numero degli elementi che compongono l'espressione (ma non per la loro lunghezza).Il dettato della versione italiana del Regolamento europeo si è trasferito nella legislazione nazionale. Parla di violazione dei dati personali, per esempio, il Decreto Legislativo I8 maggio 2018, n. 5I, che accoglie nel nostro sistema giuridico il regolamento dell'Unione europea: per esempio l'art. 26 stabilisce che "in caso di violazione di dati personali, il titolare del trattamento notifica la violazione al Garante con le modalità di cui all'articolo 33 del regolamento UE'.Molti testi ufficiali affiancano all'espressione italiana la denominazione in inglese. Si tratta di un indizio del fatto che l'anglismo data breach deve avere una certa diffusione, tra quanti si occupano della gestione dei dati personali (ma non tra $\mathrm{i}$ parlanti comuni), al punto che molti ritengono necessario esplicitare l'equivalenza tra la denominazione in italiano e quella in inglese. Adotta questa soluzione l'Autorità garante per la protezione dei dati personali, che intitola "Violazioni di dati personali (Data Breach)" la pagina del sito che si occupa dell'argomento (e in molti provvedimenti accompagna, almeno alla prima occorrenza nel testo, la formulazione italiana con la precisazione "c.d. data break"). Sporadicamente, la stessa Autorità ricorre esclusivamente all'anglismo (per esempio nella deliberazione del 6 febbraio 2020 sull'attività ispettiva curata dall'Ufficio del Garante, si legge: "limitatamente al periodo gennaiogiugno 2020, l'attività ispettiva di iniziativa curata dall'Ufficio del Garante, anche per mezzo della Guardia di finanza, è indirizzata: a) ad accertamenti in riferimento a profili di interesse generale per categorie di interessati nell'ambito di: [...] data breach").

Anche nelle discussioni parlamentari, soprattutto nelle interrogazioni più recenti, può comparire il solo anglismo. È quello che si riscontra, per fare l'esempio più recente, nell'intervento del 9 aprile 2020 alla Camera con il quale il sottosegretario alla Presidenza del Consiglio Andrea Martella ha risposto a un'interpellanza proprio sulla violazione che ha riguardato il sito dell'INPS: "va detto che il 3 aprile, proprio sul sito Internet, l'Istituto ha informato gli utenti di avere prontamente notificato il data breach al Garante per la protezione dei dati personali".

Sull'opportunità di ricorrere ai forestierismi, e in particolare agli anglismi, senza impegnarsi nella ricerca di un corrispondente italiano, ci sono comportamenti diversi e reazioni diverse, anche se si va estendendo il numero dei parlanti che finiscono per accogliere acriticamente forestierismi non adattati. Non sono mancate, però, prese di posizione e azioni per contrastare questo comportamento diffuso: la più emblematica, perché ha avuto un ampio riscontro (quasi 70.ooo adesioni in pochi giorni), è stata, nel 2015, la petizione Dillo in italiano ("Una petizione per invitare il governo italiano, le amministrazioni pubbliche, i media, le imprese a parlare un po' di più, per favore, in italiano").

Pur di fronte a posizioni diverse circa l'opportunità dell'utilizzo di forestierismi, ci dovrebbe essere un principio fondamentale, che dovrebbe essere ampiamente condiviso, soprattutto quando si tratta di testi prodotti dalle amministrazioni pubbliche: i forestierismi devono comunque garantire la comprensibilità del testo per il parlante medio (per esempio perché sono già frequentemente usati in italiano). Spesso, invece, le parole straniere sono lo strumento moderno di un vecchio vizio delle comunicazioni delle amministrazioni pubbliche: quello di essere fumose o criptiche, o comunque scarsamente comprensibili per la maggior parte della popolazione. Che sia necessario lavorare ancora alacremente per superare questo vizio capitale della comunicazione pubblica è dimostrato dall'accordo siglato il i7 febbraio 2020 tra l'Accademia della Crusca e il Ministero della Funzione Pubblica per favorire il buon uso della lingua italiana nella comunicazione tra l'amministrazione e i cittadini.

Certamente, l'uso di forestierismi di uso poco comune non favorisce la buona comunicazione tra l'amministrazione e cittadini. Spesso i forestierismi usati dall'amministrazione pubblica e dalla politica 
designano nozioni o eventi sgradevoli, critiche, divisive; nozioni, cioè, di cui ci si vergogna di parlare in maniera diretta e troppo trasparente. L'incidente accaduto alla banca dati dell'INPS, a causa del quale alcuni cittadini hanno potuto visualizzare i dati personali di altri cittadini, è un evento che risponde proprio a queste caratteristiche.

Sull'argomento specifico di data breach è intervenuto il gruppo "Incipit", attivo presso l'Accademia della Crusca, con lo scopo di monitorare i neologismi e forestierismi incipienti. Nel comunicato diffuso il 20 aprile 2020, ha suggerito di rendere l'inglese data breach con violazione dei dati, o più dettagliatamente violazione dei dati personali, cioè le espressioni già in uso in testi normativi italiani ed europei.

In conclusione, si può dare questa valutazione riassuntiva all'uso di data breach nel sito dell'INPS. Si tratta di un uso inopportuno per diversi motivi. Innanzi tutto, perché nel sito di un istituto pubblico ci si attenderebbe l'uso di espressioni italiane, se esistono. L'insensatezza di questa scelta è accresciuta dal fatto che la denominazione italiana, violazione dei dati (personali), è presente nei testi normativi, che dovrebbero essere la base delle comunicazioni di un istituto pubblico, fin quando, almeno, non si trovi un equivalente di uso più comune (ma non è certo il caso di data breach rispetto a violazione dei dati). L'uso di un forestierismo è ancora più inopportuno quando si tratta, come in questo caso, di una denominazione poco diffusa e sconosciuta a un gran numero di parlanti (e certamente sconosciuta a un gran numero di parlanti è la testa del sintagma, breach, che non compare in nessun anglismo di uso comune). L'uso di un forestierismo poco diffuso, come è il caso di data breach, è anche eticamente inopportuno, quando diventa, magari anche al di là delle intenzioni dell'estensore del testo, uno strumento per mascherare una nozione indesiderata. Infine, l'uso di un forestierismo, per di più poco trasparente, è totalmente inopportuno nel sito di un istituto che si rivolge allintera popolazione, con una particolare incidenza della quota più anziana.

\section{Cita come:}

MIchele A. Cortelazzo, Data breach o violazione dei dati? , "Italiano digitale", 2020, XIII, 2020/2 (aprile-giugno)

DOI: 10.35948/2532-9006/2020.3320

\section{Copyright 2020 Accademia della Crusca}

Pubblicato con licenza creative commons CC BY-NC-ND 